\title{
A Case of Sepsis Due to Carbapenem-Resistant Klebsiella pneumoniae in an Extremely Low-Birth Weight Infant Treated with Trimethoprim- Sulfamethoxazole
}

Bowen Weng

Xiaoyue Zhang

Wenchao Hong

Chongbing Yan

Xiaohui Gong

Cheng Cai

Department of Neonatology, Shanghai Children's Hospital, Shanghai Jiao Tong University, Shanghai, 200062, People's

Republic of China
Correspondence: Cheng Cai

Department of Neonatology, Shanghai Children's Hospital, Shanghai Jiao Tong

University, Shanghai, 200062, People's

Republic of China

Tel +862152976179

Fax +86 21 62790494

Email caicheng2004@163.com
Abstract: In recent years, there have been an increasing number of infections due to multidrug-resistant organisms in the neonatal intensive care unit. Carbapenem-resistant Klebsiella pneumoniae (CRKP) is a challenge in clinical anti-infection treatment. Herein, we report the case of CRKP sepsis in an extremely low-birth weight infant (ELBWI) who did not respond to meropenem and vancomycin, but was treated successfully after a 10-day antibiotic course with trimethoprim-sulfamethoxazole (TMP-SMZ). Recent research on CRKP-associated sepsis and the application of TMP-SMZ therapy in children and neonates were reviewed to offer a reference for clinical practice.

Keywords: carbapenem-resistance, extremely low birth weight infant, Klebsiella pneumoniae, sepsis, trimethoprim-sulfamethoxazole

\section{Introduction}

Klebsiella pneumoniae (K. pneumoniae) was first identified by Carl Friedländer in 1882. It is a lactose-fermenting, nonmotile, facultative anaerobe and Gram-negative bacillus occurring naturally in the soil. Although generally found in normal flora, it is one of the important pathogens in nosocomial infections. It causes pneumonia and sepsis in premature and newborn infants and in immunocompromised patients. ${ }^{1}$

Neonatal sepsis accounts for $30-50 \%$ of the annual neonatal deaths in developing countries. ${ }^{2} \mathrm{~K}$. pneumoniae $(17.54 \%)$ is one of the most prevalent causative bacterial pathogens in neonatal sepsis. ${ }^{2}$ Carbapenem-resistance poses a serious challenge in clinical treatment. The incidence rate of carbapenem-resistant gramnegative late-onset sepsis (LOS) was 6.5 cases per 1000 patient-days, and the most frequently isolated bacterial strain was $K$. pneumoniae. ${ }^{3}$ Carbapenem-resistant K. pneumoniae (CRKP) is associated with long hospitalizations and poor outcomes, and failure to recognize CRKP septicemia early and determine the optimum antibiotic therapy may have fatal consequences. ${ }^{4}$ However, because of concerns regarding the safety and efficacy of antibiotic therapy in the pediatric population, there were few appropriate antibiotics for CRKP treatment.

In this study, we report the case of CRKP sepsis in an extremely low-birth weight infant (ELBWI) who did not respond to meropenem and vancomycin, but was treated successfully after a 10-day antibiotic course with trimethoprim- 
sulfamethoxazole (TMP-SMZ). The parents of the patient provided informed consent for the case details to be published. Shanghai Children's Hospital approval to publish the case details.

\section{Case Report}

A premature male infant of $28^{+1}$ weeks gestation was born vaginally to a mother with a triplet pregnancy and premature rupture of membranes around $89 \mathrm{~h}$ in People's Hospital of Putuo District on May 14, 2020. The amniotic fluid, placenta, and umbilical cord were normal. This infant was transported to neonatal intensive care unit (NICU) of Shanghai Children's Hospital because of tachypnea and grunting at 1 hour postnatally. The birthweight was $970 \mathrm{~g}$, and the 1-, 5-, and 10-min Apgar scores were 3,6 , and 9 , respectively. The vital signs were stable in conventional mechanical ventilation at admission.

On day 8 of admission, he had fever and frequent apnea; by day 15 , he had fever with lung exudation on radiography and the usual infection parameters. On day 23, he developed symptoms of necrotizing enterocolitis (NEC), and the C-reactive protein (CRP) was $74 \mathrm{mg} / \mathrm{L}$. By day 45 , he deteriorated rapidly with increased liver enzymes (serum alanine aminotransferase (ALT), $145 \mathrm{U} / \mathrm{L}$ ), thrombocytopenia, and anemia. Central nervous system infection was excluded by lumbar puncture. Cultures from tracheal intubation, sputum, and blood all showed the same CRKP strain growth. In vitro susceptibility test showed that this strain was only sensitive to ceftazidime avibactam (DISK 26mm), polymyxin B (DISK 16mm), TMP-SMZ (minimal inhibitory concentrations $(\mathrm{MIC}) \leq 20 \mathrm{ug} / \mathrm{mL}$ ) and tigecycline (MIC $2 \mathrm{ug} / \mathrm{mL}$ ). After anti-infection treatment, CRP was significantly reduced on day 49 , with gradual resolution of thrombocytopenia and anemia. However, serum ALT was $345 \mathrm{U} / \mathrm{L}$ on day 51 and $878 \mathrm{U} / \mathrm{L}$ on day 58. Repeat blood culture showed negative on day 69 , but the sputum culture was still K. pneumoniae positive. The patient's liver function returned to normal on day 85 . Unfortunately, he developed bronchopulmonary dysplasia (BPD) and retinopathy of prematurity (ROP). The patient was eventually discharged on day 87 .

Regarding treatment, the patient needed mechanical ventilation for 4 days followed by 10 days of nasal continuous positive airway pressure (NCPAP). He was administered ampicillin sulbactam after admission, and ceftazidime was used for empirical therapy on day 8 , which was changed to sulperazone on day 15 . He underwent emergency abdominal drainage surgery and was administered meropenem after developing NEC on day
23; vancomycin (15 mg/kg, ivgtt, $\mathrm{q} 12 \mathrm{~h}$ ) along with mechanical ventilation was required when he deteriorated rapidly on day 45. According to the clinical symptoms and in vitro susceptibility test, we discontinued vancomycin and switched to TMP-SMZ ( $20 \mathrm{mg} / \mathrm{kg}$, twice daily) for 10 days. The patient eventually got better, and the mechanical ventilation was switched to NCPAP on day 51, and oxygen was discontinued from day 75 onward. Caffeine treatment was started from the first day after birth and was maintained for up to 63 days. The patient received partial parenteral venous nutrition before complete enteral feeding, compound glycyrrhizin and coenzyme complex injections for abnormal liver function, and oral diuretics and inhalant budesonide as treatments for BPD.

\section{Discussion}

This report describes a case of CRKP sepsis in an ELBWI who required surgery for NEC that was only sensitive to ceftazidime avibactam, polymyxin B, TMP-SMZ, and tigecycline. K. pneumoniae can cause life-threatening hospital acquired bloodstream infections (BSI), with a high risk of mortality of $54.3 \% .{ }^{5}$ Unfortunately, it is difficult to choose the appropriate antibiotic to treat K. pneumoniae as there is no licensed antibiotic available to treat it in China until now. ${ }^{6}$ In this case, the infant got worse after treatment with meropenem and vancomycin, so we administered TMP-SMZ for 10 days based on the results of the antimicrobial susceptibility assay. The infant was discharged after his condition gradually improved. To our knowledge, this is the first and only case of CRKP sepsis in an ELBWI who was treated with TMP-SMZ in our NICU.

In a study of healthcare-associated BSI, $K$. pneumoniae comprised the largest proportion of pathogenic organisms in healthcare-associated BSI (32\%, 93/292). Furthermore, in a subset of 12 neonates with $K$. pneumoniae bacteremia, it was observed that the median (interquartile range) gestational age at birth was 27 weeks, and the median birth weight was 1100 g. $^{7}$ The 28-day mortality of K. pneumoniae BSI was $8.7 \% .{ }^{5}$ In our patient, the infant had high risk factors for CRKP infection, such as mechanical ventilation, total parenteral intravenous nutrition support, long-term use of broad-spectrum antimicrobial agents, and admission to the NICU. In addition, the K. pneumoniae was only sensitive to ceftazidime avibactam, polymyxin B, TMP-SMZ, and tigecycline. It has been reported that an infant with CRKP invasive infections required ceftazidime avibactam (CAZ-AVI), phosphomycin, and meropenem as a combination treatment. ${ }^{8}$ However, this patient had not only 
moderate thrombocytopenia during the therapy but also recurrent CRKP sepsis after the antibiotics were discontinued for 2 days. ${ }^{8}$ In a study of colistin use in neonatal sepsis, only one of the seven patients with sepsis due to K. pneumoniae survived. Furthermore, there were no pharmacokinetic and pharmacodynamic studies performed with colistin in neonatal sepsis. ${ }^{9}$ There are many limitations of tigecycline treatment in pediatric patients, for example, the reported dosage and treatment duration were varied with small sample size, and it is often used in combination with other antimicrobial agents (Table 1). ${ }^{10}$

Neonatal sepsis remains a major cause of death in very low birth weight infants with high risk for both short-term complications, such as BPD, NEC, intraventricular hemorrhage, and periventricular leukomalacia and long-term complications including cerebral palsy, psychomotor delay, and visual and auditory impairment. ${ }^{11} \mathrm{~K}$. pneumoniae is one of the top three isolated pathogens in neonatal sepsis. Patients infected with $K$. pneumoniae have a high incidence of thrombocytopenia. ${ }^{6}$ Our patient developed thrombocytopenia, BPD, and ROP after being diagnosed with sepsis. The blood platelet count returned to normal after TMP-SMZ antibiotic therapy for 3 days, and supplemental oxygen was discontinued at postnatal 75 days. The ROP showed gradual resolution at the follow-up fundus examination.
TMP-SMZ is one of the few remaining antimicrobial agents with some activity against CRKP in China. ${ }^{12}$ However, the functions of the liver and kidney in children are immature, and the acetyltransferase system in neonates has not yet developed. Therefore, the TMP-SMZ can compete with the bilirubin content in the plasma protein-binding site, which causes the blood concentration of free sulfanilamide to increase. This, in turn, increases the risks of kernicterus; hence, the use of TMP-SMZ in children and infants has its limitations. In another case report, a premature infant who developed sepsis, meningitis, and hydrocephalus was treated successfully with TMP-SMZ for 3 weeks with no side effects. ${ }^{13}$ Therefore, although the state drug administration has not approved TMP-SMZ use in infants younger than 2 months, in the absence of safe and effective alternatives, TMP-SMZ may be used as a reasonable substitute in order to reduce the mortality from CRKP sepsis in ELBWI. Our patient had elevated liver enzymes, which is a known associated side effect; nonetheless, with symptomatic treatment, the patient's liver function returned to normal on day 85 . We recommended that liver and kidney function should be followed up at least once a week in infants treated with TMPSMZ. Moreover, we also carried out follow-up magnetic resonance imaging of the head to monitor any adverse reactions to TMP-SMZ since the discharge.

Table I The Usage of Antimicrobials and Efficacy in Cases of Neonatal Sepsis

\begin{tabular}{|c|c|c|c|c|}
\hline $\begin{array}{l}\text { Author (Year } \\
\text { of } \\
\text { Publication) }\end{array}$ & Pathogen & $\begin{array}{c}\text { Usage of } \\
\text { Antimicrobials }\end{array}$ & Efficacy & Side Effects \\
\hline $\begin{array}{l}\text { Shobowale } \\
\text { et al }(2017)^{14}\end{array}$ & CONS and $K$. Pneumoniae & $\begin{array}{l}\text { Meropenem, } \\
\text { ciprofloxacin, and } \\
\text { amikacin }\end{array}$ & Most effective antimicrobials in vitro & I \\
\hline $\begin{array}{l}\text { Esposito et al } \\
(2019)^{8}\end{array}$ & CRKP & $\begin{array}{c}\text { CAZ-AVI/ } \\
\text { phosphomycin/ } \\
\text { meropenem combination }\end{array}$ & Recurrent CRKP sepsis & $\begin{array}{l}\text { Moderate } \\
\text { thrombocytopenia }\end{array}$ \\
\hline $\begin{array}{l}\text { Jasani et al } \\
(2016)^{9}\end{array}$ & $\begin{array}{l}\text { Acinetobacter baumannii, } \\
\text { K. Pneumoniae and Pseudomonas } \\
\text { aeruginosa }\end{array}$ & Intravenous colistin & $\begin{array}{l}\text { Only one of seven with } K . \text { Pneumoniae } \\
\text { infection survived }\end{array}$ & $\begin{array}{l}\text { None } \\
\text { neurotoxicity or } \\
\text { nephrotoxicity }\end{array}$ \\
\hline $\begin{array}{l}\text { Su et al } \\
(2019)^{12}\end{array}$ & CRKP & $\begin{array}{l}\text { TMP-SMZ / colistin } \\
\text { combination }\end{array}$ & Effective in Vitro & I \\
\hline $\begin{array}{l}\text { Gokce et al } \\
(2012)^{13}\end{array}$ & Elizabethkingia & TMP-SMZ for 3 weeks & $\begin{array}{l}\text { CSF examination returned to normal, } \\
\text { but the patient developed } \\
\text { hydrocephalus }\end{array}$ & $\begin{array}{l}\text { No side-effects } \\
\text { occurred }\end{array}$ \\
\hline
\end{tabular}

Abbreviations: CONS, coagulase-negative staphylococci; K. pneumoniae, Klebsiella pneumoniae; CRKP, Carbapenem-resistant Klebsiella pneumoniae; CAZ-AVI, ceftazidime avibactam; TMP-SMZ, trimethoprim-sulfamethoxazole; CSF, Cerebrospinal fluid. 
In summary, this case report describes CRKP sepsis, which is associated with high morbidity and mortality, in an ELBWI. We believe that timely and rational therapy with TMP-SMZ in these premature infants may reduce mortality and improve prognosis of CRKP sepsis.

\section{Abbreviations}

NICU, neonatal intensive care unit; CRKP, carbapenemresistant Klebsiella pneumoniae; ELBWI, extremely low birth weight infant; TMP-SMZ, trimethoprim-sulfamethoxazole; K. pneumoniae, Klebsiella pneumoniae; LOS, late-onset sepsis; NRDS, neonatal respiratory distress syndrome; NEC, neonatal necrotizing enterocolitis; CSF, Cerebrospinal fluid; BPD, bronchopulmonary dysplasia; ROP, retinopathy of prematurity; NCPAP, nasal continuous positive airway pressure; BSI, bloodstream infections; CAZ-AVI, ceftazidime avibactam.

\section{Data Sharing Statement}

Not shared as it contains confidential patient data.

\section{Ethics Approval and Informed Consent}

The parents of the patient provided informed consent for the case details to be published. Shanghai Children's Hospital approval to publish the case details.

\section{Consent for Publication}

The parents of this patient provided the written permission to the publication.

\section{Author Contributions}

WBW, CC, and HWC made substantial contributions to the conception and design, and the acquisition, analysis, and interpretation of data. WBW, ZXY, YCB, and CC were involved in drafting the manuscript and revising it critically for important intellectual content. CC and GXH revised the manuscript and gave the final approval for the version to be published. All authors contributed to data analysis, drafting or revising the article, have agreed on the journal to which the article will be submitted, gave final approval for the version to be published, and agree to be accountable for all aspects of the work.

\section{Disclosure}

All authors certify that they have no affiliations with or involvement in any organization or entity with any financial interest, or non-financial interest in the subject matter or materials discussed in this manuscript.

\section{References}

1. Bengoechea JA, Sa Pessoa J. Klebsiella pneumoniae infection biology: living to counteract host defences. FEMS Microbiol Rev. 2019;43(2):123-144.

2. Akbarian-Rad Z, Riahi SM, Abdollahi A, et al. Neonatal sepsis in Iran: a systematic review and meta-analysis on national prevalence and causative pathogens. PLoS One. 2020;15(1):e0227570. doi:10.1371/journal.pone. 0227570

3. Nour I, Eldegla HE, Nasef N, Shouman B, Abdel-Hady H, Shabaan AE. Risk factors and clinical outcomes for carbapenem-resistant Gram-negative late-onset sepsis in a neonatal intensive care unit. J Hosp Infect. 2017;97(1):52-58. doi:10.1016/j. jhin.2017.05.025

4. Di Tella D, Tamburro M, Guerrizio G, Fanelli I, Sammarco ML, Ripabelli G. Molecular epidemiological insights into colistin-resistant and carbapenemases-producing clinical Klebsiella pneumoniae isolates. Infect Drug Resist. 2019;12:3783-3795. doi:10.2147/IDR. S226416

5. Zhang Y, Guo LY, Song WQ, Wang Y, Dong F, Liu G. Risk factors for carbapenem-resistant $\mathrm{K}$. pneumoniae bloodstream infection and predictors of mortality in Chinese paediatric patients. BMC Infect Dis. 2018;18(1):248. doi:10.1186/s12879-018-3160-3

6. Guo J, Luo Y, Wu Y, Lai W, Mu X. Clinical characteristic and pathogen spectrum of neonatal sepsis in Guangzhou City from June 2011 to June 2017. Med Sci Monit. 2019;25:2296-2304. doi:10.12659/MSM.912375

7. Essel V, Tshabalala K, Ntshoe G, et al. A multisectoral investigation of a neonatal unit outbreak of Klebsiella pneumoniae bacteraemia at a regional hospital in Gauteng Province, South Africa. $S$ Afr Med J. 2020;110(8):783-790. doi:10.7196/SAMJ.2020.v110i8.14471

8. Esposito P, Sbrana F, Di Toro A, Gombos S, Tascini C. Ceftazidineavibactam salvage therapy in newborn with KPC-producing Klebsiella pneumoniae invasive infections. Minerva Anestesiol. 2019;85(7):804-805. doi:10.23736/S0375-9393.19.13521-3

9. Jasani B, Kannan S, Nanavati R, Gogtay NJ, Thatte U. An audit of colistin use in neonatal sepsis from a tertiary care centre of a resource-limited country. Indian J Med Res. 2016;144(3):433-439. doi:10.4103/0971-5916.198682

10. Mastrolia MV, Galli L, De Martino M, Chiappini E. Use of tigecycline in pediatric clinical practice. Expert Rev Anti Infect Ther. 2017;15(6):605-612. doi:10.1080/14787210.2017.1318064

11. Bakhuizen SE, de Haan TR, Teune MJ, et al. Meta-analysis shows that infants who have suffered neonatal sepsis face an increased risk of mortality and severe complications. Acta Paediatr. 2014;103 (12):1211-1218. doi:10.1111/apa.12764

12. Su J, Li D, Guo Q, Guo Y, Zheng Y, Xu X. In vitro bactericidal activity of trimethoprim-sulfamethoxazole/colistin combination against carbapenem-resistant Klebsiella pneumoniae clinical isolates. Microb Drug Resist. 2019;25(2):152-156. doi:10.1089/ mdr.2018.0085

13. Gokce IK, Oncel MY, Ozdemir R, et al. Trimethoprimsulfamethoxazole treatment for meningitis owing to multidrug-resistant Elizabethkingia meningoseptica in an extremely low-birthweight, premature infant. Paediatr Int Child Health. 2012;32(3):177-179. doi:10.1179/2046905511Y.0000000008

14. Shobowale EO, Solarin AU, Elikwu CJ, Onyedibe KI, Akinola IJ, Faniran AA. Neonatal sepsis in a Nigerian private tertiary hospital: bacterial isolates, risk factors, and antibiotic susceptibility patterns. Ann Afr Med. 2017;16(2):52-58. doi:10.4103/aam. aam_34_16 


\section{Publish your work in this journal}

Infection and Drug Resistance is an international, peer-reviewed openaccess journal that focuses on the optimal treatment of infection (bacterial, fungal and viral) and the development and institution of preventive strategies to minimize the development and spread of resistance. The journal is specifically concerned with the epidemiology of antibiotic resistance and the mechanisms of resistance development and diffusion in both hospitals and the community. The manuscript management system is completely online and includes a very quick and fair peerreview system, which is all easy to use. Visit http://www.dovepress.com/ testimonials.php to read real quotes from published authors. 